\title{
Thin film models in a stochastic setting
}

\author{
T. Prvan* M. R. Osborne
}

(Received 2 December 2004, revised 2 May 2005)

\begin{abstract}
Dunn \& Tichenor (1988) proposed a class of differential equation models to describe the phenomenon of transient sink behaviour for organic emissions exhibited by interior surface films in state-of-the-art emission test chambers. Data from a particular application is used to exemplify the use of a model selection scheme which embeds the derived models within a class of stochastic differential equations. These embeddings have the property that the quality of model fit varies inversely with the strength of the stochastic forcing term. Results of this modelling application are discussed.
\end{abstract}

\section{Contents}

\section{General form of thin film models}

${ }^{*}$ Department of Statistics, Macquarie University, Sydney, N.S.W. 2109 Australia. mailto:tania. prvan@mq.edu . au

${ }^{\dagger}$ Mathematical Sciences Institute, Australian National University, A.C.T. 0200, AustraliA. mailto:Mike.Osborne@maths.anu .edu. au

See http://anziamj.austms.org.au/V46/CTAC2004/Prva for this article, (c) Austral. Mathematical Soc. 2005. Published June 15, 2005. ISSN 1446-8735 
2 Thin film models in a stochastic setting

2.1 Stochastic formulation of smoothing spline . . . . . . . C508

2.2 Stochastic formulation of thin film models . . . . . . . C509

3 Moth crystal cake example

C512

3.1 Dilution model $\left(k_{3}=0, k_{5}=0\right) \ldots \ldots \ldots \ldots$ C512

References

C515

\section{General form of thin film models}

Dunn and Tichenor [1] present evidence that interior surface films in stateof-the art emission chambers acts as a transient sink for organic emissions. They developed a class of differential equations to model this phenomenon. It is possible within their setting to consider four classes of models: full models, sink models, vapour pressure models and dilution models.

Dunn and Tichenor [1] visualise a test chamber system consisting of four compartments: the source, the well mixed chamber contents, an exit, and a sink. The source is a film source which may be decreasing (for example, carpet glue on an inert carrier) or constant (for example, a cake of moth crystal). The following diagram describes the situation being considered.

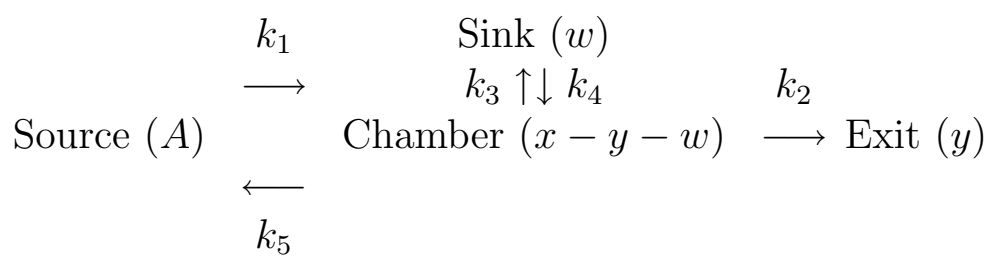

The initial mass emitted by the source is denoted by $A$; the mass emitted to the chamber by time $t$ is denoted by $x=x(t)$; the mass exiting the chamber by time $t$ is denoted by $y=y(t)$; the mass in the sink at time $t$ 
Models

\section{TABLE 1:}

\begin{tabular}{lll}
\hline Full & $k_{3} \neq 0$ & $k_{5} \neq 0$ \\
Sink & $k_{3} \neq 0$ & $k_{5}=0$ \\
Vapour pressure & $k_{3}=0$ & $k_{5} \neq 0$ \\
Dilution & $k_{3}=0$ & $k_{5}=0$ \\
\hline
\end{tabular}

is denoted by $w=w(t)$; and the concentration in the chamber at time $t$ is $C(t)=(x-y-w) / V$ where $V$ is the chamber volume.

In a well mixed chamber Dunn and Tichenor [1] describe the system by

$$
\begin{aligned}
\frac{d x}{d t} & =k_{1} g(x, t)-k_{5}(x-y-w), \\
\frac{d y}{d t} & =k_{2}(x-y-w), \\
\frac{d w}{d t} & =k_{3}(x-y-w)-k_{4} w .
\end{aligned}
$$

Assuming a constant air rate $F$ through the chamber we have that $k_{2}=F / V$, the number of air changes in unit time. This is fixed by the experimental set up. The full set of models possible in this setting are summarized in Table 1.

In particular, we are interested in constant source models. Here the source acts as if it were a constant emitter for a finite period of time. For example, the moth crystal cake has its emission rate limited by its surface area, so effectively $A=\infty$; and if potential for emissions is constant during the time period considered, then $g(x, t)=1$ in equation (1).

\section{Thin film models in a stochastic setting}

First we briefly look at the stochastic formulation of the smoothing spline and extend this to fit thin film models in a stochastic setting. 


\subsection{Stochastic formulation of smoothing spline}

Suppose that the data $\left(t_{1}, y_{1}\right), \ldots,\left(t_{n}, y_{n}\right)$ are given and it is assumed that the data is decomposed as the signal plus noise model

$$
y_{i}=f\left(t_{i}\right)+\epsilon_{i}, \quad \epsilon_{i} \sim N\left(0, \sigma^{2}\right), \quad i=1, \ldots, n .
$$

We could use a smoothing spline to fit $f(t)$. A smoothing spline $f$ is the minimizer of

$$
\sum_{i=1}^{n}\left(y_{i}-f\left(t_{i}\right)\right)^{2}+\mu \int_{t_{1}}^{t_{n}}\left(f^{(m)}(t)\right)^{2} d t .
$$

The resultant curve is a piecewise polynomial of degree $2 m-1$ with $2 m-2$ continuous derivatives.

Wahba [4] showed that a polynomial smoothing spline is the solution to the stochastic differential equation

$$
\frac{d^{m} x}{d t^{m}}=\sigma \sqrt{\lambda} \frac{d \omega}{d t}
$$

where $\omega(t)$ is a Wiener process with unit dispersion parameter, $\lambda=1 / \mu$, and $\boldsymbol{x}\left(t_{1}\right)=\left[\begin{array}{llll}x\left(t_{1}\right) & x^{\prime}\left(t_{1}\right) & \cdots & x^{(m-1)}\left(t_{1}\right)\end{array}\right]^{T}$ has a diffuse prior (that is, $\boldsymbol{x}\left(t_{1}\right) \sim N\left(\mathbf{0}, \gamma^{2} I_{m}\right)$ and $\left.\gamma^{2} \rightarrow \infty\right)$.

Wecker and Ansley [5] presented a stochastic formulation of a polynomial smoothing spline using this result.

Rewrite the stochastic differential equation (6) as

$$
\frac{d \boldsymbol{x}}{d t}=\left(\begin{array}{cc}
\mathbf{0}_{m-1} & I_{m-1} \\
0 & \mathbf{0}^{T}
\end{array}\right) \boldsymbol{x}+\sigma \sqrt{\lambda} \frac{d \omega}{d t} \boldsymbol{e}_{m},
$$

and the observation equation (4) as

$$
y\left(t_{i}\right)=\boldsymbol{e}_{1}^{T} \boldsymbol{x}\left(t_{i}\right)+\epsilon_{i} .
$$




\subsection{Stochastic formulation of thin film models}

We generalise the stochastic differential equation for smoothing splines to

$$
\frac{d \boldsymbol{x}}{d t}=M \boldsymbol{x}+\boldsymbol{g}(t)+\sigma \sqrt{\lambda} \frac{d \omega}{d t} \boldsymbol{b},
$$

where $M: \mathbb{R}^{m} \rightarrow \mathbb{R}^{m}$ and $\omega(t)$ is a Wiener process with unit dispersion parameter. The initial conditions are the same as for the polynomial smoothing spline and point estimates are $E\left(\boldsymbol{h}^{T} \boldsymbol{x}(t) \mid y_{1}, \ldots, y_{n}\right)$. The corresponding observation equation is

$$
y_{i}=\boldsymbol{h}^{T} \boldsymbol{x}\left(t_{i}\right)+\epsilon_{i} .
$$

If, when fitting this "extended" generalised smoothing spline, $\lambda$ tends to be small, then the model is adequate. Note that the scale of the observation has been factored out of $\lambda$ in (9).

We now obtain the state space formulation of the "extended" generalized smoothing spline. Let $X\left(t, t_{1}\right)$ be the fundamental matrix solution of the associated homogeneous differential equation. That is,

$$
\frac{d X}{d t}=M X, \quad X\left(t_{1}, t_{1}\right)=I_{m}
$$

The solution to the stochastic differential equation (9) satisfying $\boldsymbol{x}\left(t_{1}\right)=\boldsymbol{x}_{1}$ is

$$
\boldsymbol{x}(t)=X\left(t, t_{1}\right) \boldsymbol{x}_{1}+\int_{t_{1}}^{t} X(t, s) \boldsymbol{g}(s) d s+\sigma \sqrt{\lambda} \int_{t_{1}}^{t} X(t, s) \boldsymbol{b} \frac{d \omega}{d s} d s .
$$

This solution can be written in the form of a recursion as

$$
\boldsymbol{x}_{i+1}=X_{i+1} \boldsymbol{x}_{i}+\int_{t_{i}}^{t_{i+1}} X\left(t_{i+1}, s\right) \boldsymbol{g}(s) d s+\sigma \sqrt{\lambda} \boldsymbol{u}_{i+1},
$$


with $\boldsymbol{x}_{i}=\boldsymbol{x}\left(t_{i}\right), X_{i+1}=X\left(t_{i+1}, t_{i}\right)$ and $\boldsymbol{u}_{i+1}=\boldsymbol{u}\left(t_{i+1}, t_{i}\right)$ where $\boldsymbol{u}_{i+1}=$ $\int_{t_{i}}^{t_{i+1}} X\left(t_{i+1}, s\right) \boldsymbol{b} \frac{d \omega}{d s} d s$ which has a $N\left(\mathbf{0}, \Omega\left(t_{i+1}, t_{i}\right)\right)$ distribution with covariance matrix

$$
\Omega_{i+1}=\Omega\left(t_{i+1}, t_{i}\right)=\int_{t_{i}}^{t_{i+1}} X\left(t_{i+1}, s\right) \boldsymbol{b} \boldsymbol{b}^{T} X\left(t_{i+1}, s\right)^{T} d s .
$$

We now have the state space formulation (10) and (13). We obtain $\boldsymbol{x}(t \mid n)$ and hence the point estimate $\boldsymbol{h}^{T} \boldsymbol{x}(t \mid n)$ by

1. forward pass of the Kalman filter for state equation with forcing term (which exists in the literature),

2. followed by a backward pass of the RTS smoother (that in the literature still applies)

3. then an application of an interpolation smoother modified for state equation with forcing term (this is new).

Steps 1 and 2 need only be done once for fixed $\lambda$. Maximum Likelihood Estimation (MLE) or Generalized Cross Validation (GCV) can be used to find the smoothing parameter. For reference the recursions are given below.

Kalman Filter Initiate with $x_{1 \mid 0}=\mathbf{0}$ and $S_{1 \mid 0}=\gamma^{2} I_{m}$ :

$$
\begin{aligned}
\boldsymbol{x}_{k+1 \mid k}= & X_{k+1} \boldsymbol{x}_{k \mid k}+\int_{t_{k}}^{t_{k+1}} X\left(t_{k+1}, s\right) \boldsymbol{g}(s) d s, \\
S_{k+1 \mid k}= & X_{k+1} S_{k+1 \mid k} X_{k+1 \mid k}^{T}+\lambda \sigma^{2} \Omega\left(t_{k+1}, t_{k}\right), \\
d_{k+1}= & \boldsymbol{h}^{T} S_{k+1 \mid k} \boldsymbol{h}+\sigma^{2} \\
\boldsymbol{x}_{k+1 \mid k+1}= & \boldsymbol{x}_{k+1 \mid k}+S_{k+1 \mid k} \boldsymbol{h} d_{k+1}^{-1}\left(y_{k+1}-\boldsymbol{h}^{T} \boldsymbol{x}_{k+1 \mid k}\right), \\
S_{k+1 \mid k}= & S_{k+1 \mid k}-S_{k+1 \mid k} \boldsymbol{h} d_{k+1}^{-1} \boldsymbol{h}^{T} S_{k+1 \mid k}^{T}, \\
& k=1, \ldots, n-1 .
\end{aligned}
$$


RTS Smoother Initiate with $\boldsymbol{x}_{n \mid n}$ obtained from a forward pass of the Kalman Filter:

$$
\begin{aligned}
\boldsymbol{x}_{k \mid n}= & \boldsymbol{x}_{k \mid k}+S_{k \mid k} X_{k+1}^{T} S_{k+1 \mid k}^{-1}\left(\boldsymbol{x}_{k+1 \mid n}-\boldsymbol{x}_{k+1 \mid k}\right), \\
& k=n, n-1, \ldots, 1 .
\end{aligned}
$$

Interpolation Smoother For $t_{i-1} \leq t<t_{i}$ :

$\boldsymbol{x}(t \mid n)=X\left(t, t_{i-1}\right) \boldsymbol{x}_{i-1 \mid i-1}+\int_{t_{i-1}}^{t} X(t, s) \boldsymbol{g}(s) d s+A\left(t_{i}, t\right)\left(\boldsymbol{x}_{i \mid n}-\boldsymbol{x}_{i \mid i-1}\right)$,

where

$A\left(t_{i}, t\right)=\left[X\left(t, t_{i-1}\right) S_{i-1 \mid i-1} X_{i}^{T}+\Omega\left(t, t_{i-1}\right) X\left(t_{i}, t\right)^{T}\right] S_{i \mid i-1}^{-1}$.

Smoothness properties for our "extended" generalized smoothing spline follow a similar argument to that found in Osborne and Prvan $[2,3]$.

Proposition 1 The first $k$ derivatives of $\boldsymbol{x}(t \mid n)$ are continuous if

$$
\boldsymbol{b}^{T} P_{j}(M)^{T} \boldsymbol{h}=0, \quad j=0, \ldots, k-1,
$$

where for $i=1,2, \ldots$,

$$
P_{0}(M)=I_{m}, \quad P_{i}(M)=\frac{d P_{i-1}}{d t}-M P_{i-1} .
$$

If $M$ is a constant matrix, then $P_{i}(M)=(-1)^{i} M^{i}$.

For the thin film model considered we have

$$
M=\left[\begin{array}{ccc}
-k_{5} & k_{5} & k_{5} \\
k_{2} & -k_{2} & -k_{2} \\
k_{3} & -k_{3} & -\left(k_{3}+k_{4}\right)
\end{array}\right],
$$

and $\boldsymbol{g}(t)=k_{1} \boldsymbol{e}_{1}$. We observe $y$ so $\boldsymbol{h}=\boldsymbol{e}_{2}$. 


\section{Moth crystal cake example}

Dunn and Tichenor [1] present results for 13 emissions tests of moth crystal cakes. The chamber was cleaned between each test. They fitted the four constant source models to each data set using nonlinear least squares to determine the unknown parameters in the experiment, such as $k_{1}$. The parameter $k_{2}$ is fixed by the experiment and is the the number of air changes per hour. They had $V=166 l$. The criteria they use to determine the best model for each test is to choose the model that produces the smallest residual sum of squares. We look at the test labeled 10.1 in more detail.

\subsection{Dilution model $\left(k_{3}=0, k_{5}=0\right)$}

To fit the "extended" generalized smoothing spline for this particular model we have

$$
M=\left[\begin{array}{ccc}
0 & 0 & 0 \\
k_{2} & -k_{2} & -k_{2} \\
0 & 0 & -k_{4}
\end{array}\right]
$$

and

$$
\int_{t_{i}}^{t} X(t, s) \boldsymbol{g}(s) d s=k_{1} \int_{t_{i}}^{t} X(t, s) \boldsymbol{e}_{1} d s=k_{1}\left[\begin{array}{c}
\left(t-t_{i}\right) \\
\left(t-t_{i}\right)+\frac{1}{k_{2}}\left(e^{-k_{2} t}-1\right) \\
0
\end{array}\right] .
$$

Also

$$
X\left(t, t_{i}\right)=\left[\begin{array}{ccc}
1 & 0 & 0 \\
1-e^{-k_{2}\left(t-t_{i}\right)} & e^{-k_{2}\left(t-t_{i}\right)} & \frac{k_{2}}{k_{4}-k_{2}}\left(e^{-k_{4}\left(t-t_{i}\right)}-e^{-k_{2}\left(t-t_{i}\right)}\right) \\
0 & 0 & e^{-k_{4}\left(t-t_{i}\right)}
\end{array}\right] .
$$

The choice of $\boldsymbol{h}$ is fixed by our model, we observe $y$, so $\boldsymbol{h}=\boldsymbol{e}_{2}$. It is not difficult to show that with this choice both $k_{2}$ and $k_{4}$ are observable. We 
still need to make a choice about $\boldsymbol{b}$. Using Proposition 1 we have at least one continuous derivative if $\boldsymbol{b}^{T} \boldsymbol{e}_{2}=0$ which meas that $\boldsymbol{b}=\boldsymbol{e}_{1}$ or $\boldsymbol{b}=\boldsymbol{e}_{3}$ or a linear combination of $\boldsymbol{e}_{1}$ and $\boldsymbol{e}_{3}$. To have two continuous derivatives we need that $\boldsymbol{b} M^{T} \boldsymbol{h}=0$. Looking at

$$
\begin{aligned}
\boldsymbol{b} M^{T} \boldsymbol{h} & =\left[\begin{array}{lll}
b_{1} & 0 & b_{2}
\end{array}\right]\left[\begin{array}{ccc}
0 & k_{2} & 0 \\
0 & -k_{2} & 0 \\
0 & -k_{2} & -k_{4}
\end{array}\right]\left[\begin{array}{l}
0 \\
1 \\
0
\end{array}\right] \\
& =\left[\begin{array}{lll}
b_{1} & 0 & b_{2}
\end{array}\right]\left[\begin{array}{c}
k_{2} \\
0 \\
-k_{2}
\end{array}\right]
\end{aligned}
$$

which will only equal zero if $b_{1}=b_{2}$. Without loss of generality assume that $\boldsymbol{b}=\boldsymbol{e}_{1}+\boldsymbol{e}_{3}$. We also need to obtain the state transition covariance matrix $\lambda \sigma^{2} \Omega\left(t, t_{i}\right)$ where

$$
\Omega\left(t, t_{i}\right)=\int_{t_{i}}^{t} X(t, s) \boldsymbol{b} \boldsymbol{b}^{T} X(t, s)^{T} d s .
$$

A preliminary computation has been made to evaluate the Dilution Model fitted to 1,4-Dichlorobenzene emission from moth crystal. Figure 1 shows the fit where $k_{2}=0.25, k_{1}=211, k_{4}=0.0802$ and the smoothing parameter chosen by maximum likelihood estimation is $\lambda=2.7331 \times 10^{4}$. This large smoothing parameter indicates that this model is not fitting well. We also see this from the fit in Figure 1 because the model is tending to adapt very precisely to the data. If the model fitted well, we would expect to see a smooth curve going between the points of the data and we would expect the smoothing parameter to be small.

A full study of the range of possible models is currently being undertaken. 


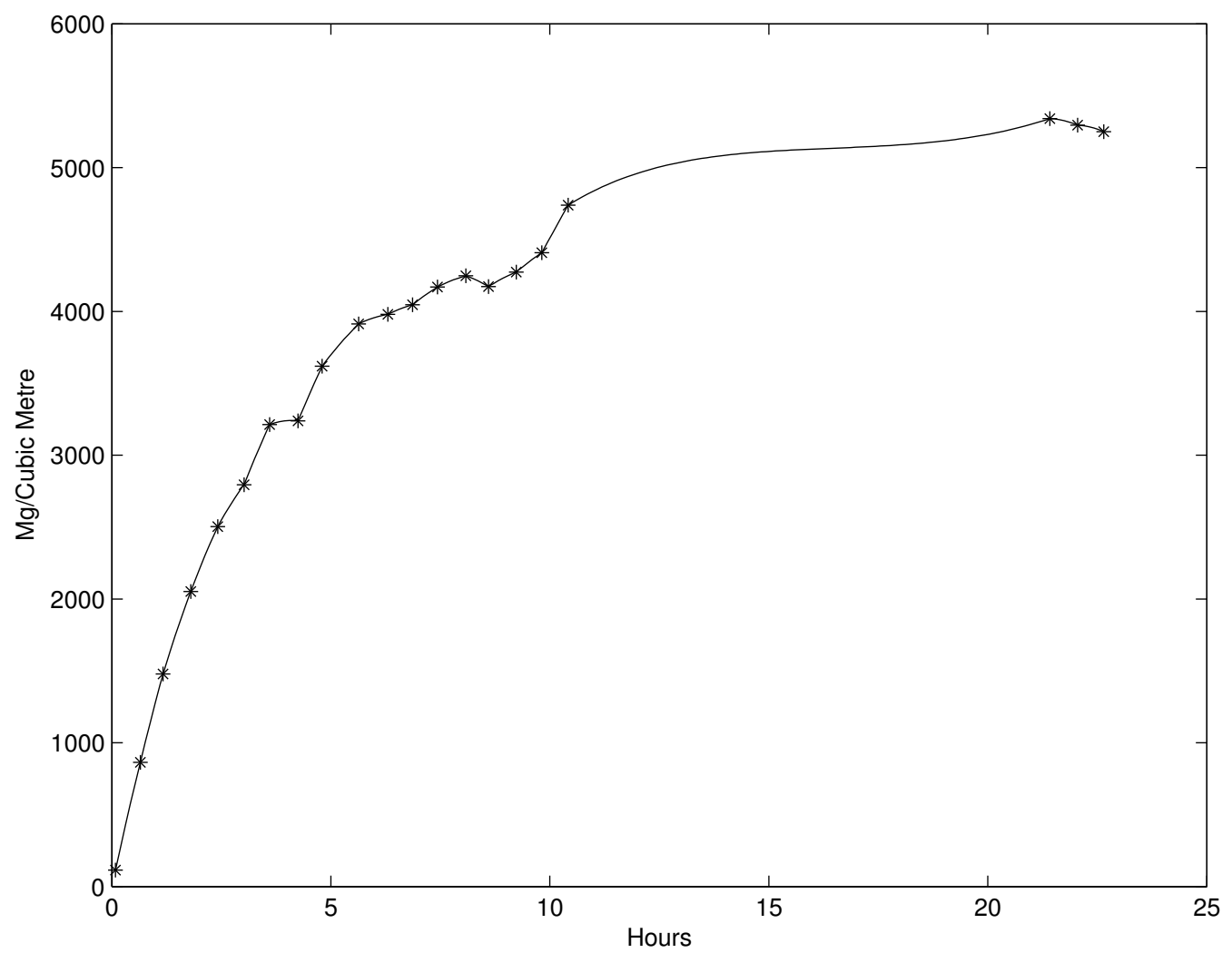

FIGURE 1: Extended generalized smoothing spline fit to dilution model fitted to 1,4-Dichlorobenzene emission from moth crystal for $k_{1}=211, k_{2}=0.25$, $k_{4}=0.0802$ and $\lambda=2.7331 \times 10^{4}$. 


\section{References}

[1] J. E. Dunn and B. A. Tichenor. Compensating for sink effects in emissions test chambers by mathematical modeling. Atmospheric Environment, 122:885-894, 1988. C506, C507, C512

[2] M. R. Osborne and T. Prvan. On algorithms for generalised smoothing splines. J. Austral. Math. Soc. Ser. B, 29:322-241, 1988. C511

[3] M. R. Osborne and T. Prvan. Smoothness and conditioning in generalised smoothing spline calculations. J. Austral. Math. Soc. Ser. B, 30:43-56, 1988. C511

[4] G. Wahba, Improper priors, spline smoothing and the problem of guarding against model errors in regression, J. Roy. Statist. Assoc., 40:364-372, 1978. C508

[5] W. Wecker and C. F. Ansley. Signal extraction approach to non linear regression and spline smoothing. J. Amer. Statist. Assoc., 78:81-89, 1983. C508 